\title{
Chapitre II \\ Les peintres viennois au service de Marie-Thérèse
}

Les peintres produisent, copient et imitent tout au long du règne les portraits de Marie-Thérèse réalisés à Vienne par quelques grands noms comme Meytens ou Maron. Si certains peintres sont inconnus, d'autres sont très clairement attachés à une période précise de la vie de Marie-Thérèse comme Andreas Möller qui peint Marie-Thérèse en 1727 à l'âge de dix ans, encore toute jeune archiduchesse. Parmi ces peintres, certains travaillent déjà pour Charles VI tels Johann Gottfried Auerbach, Hamilton et Meytens, d'autres ont véritablement été formés sous le règne de Marie-Thérèse comme Hickel et Maron. Martin van Meytens, avec son atelier, propose les principales images de la souveraine dès son arrivée sur le trône en 1740, comme il le faisait dès les années 1730 en sa qualité de portraitiste officiel de l'empereur Charles VI. Meytens et son atelier jouent ce rôle jusqu'à la mort de l'artiste en 1770, autrement dit pendant la majeure partie du règne de Marie-Thérèse.

Entre 1740 et 1750, les peintres les plus actifs pour la cour de Vienne sont Philipp Ferdinand von Hamilton, Peter Kobler, et bien sûr Martin van Meytens et son atelier. Durant la dernière décennie du règne entre 1770 et 1780, Anton von Maron offre l'image d'une impératrice veuve, assidue au travail. Cette pose reflète une nouvelle conception du rôle du monarque. Sous les règnes de MarieThérèse et de son corégent et empereur Joseph, les peintres de cour qui se révèlent particulièrement actifs sont Anton von Maron, Joseph Hickel, Johannes Tusch (ou Dusch), Hubert Maurer ou Johann Zollinger.

Il n'est pas faux de considérer Martin van Meytens comme le fondateur de l'image « Marie-Thérèse », qui imprègne le portrait de la souveraine pour la postérité, le peintre par excellence au service de la souveraine, de son époux et de ses enfants. À côté de ce grand artiste règne une multitude de « petits " peintres, moins connus, mais qui disposent aussi d'une certaine renommée. Les peintres de Marie-Thérèse, en particulier les peintres de cour qui travaillent pour la cour de Vienne, se réunissent dans la capitale viennoise, à la cour comme à l'Académie ${ }^{99}$.

À Vienne, Martin van Meytens n'est pas le seul peintre de réelle importance même si sa renommée et sa célébrité sont indéniables. D’autres peintres jouent également un rôle non négligeable dans l'élaboration de l'image de Marie-Thérèse. Les peintres de cour, souvent des peintres académiciens, travaillent tant pour la

$99 \mathrm{Au}$ sujet des peintres de cour voir Haupt, Das Hof- und hofbefreite Handwerk.

Ә Open Access. (c) 2022 Anne-Sophie Banakas, published by De Gruyter. (cc) BY-NC-ND This work is licensed under the Creative Commons Attribution-NonCommercial-NoDerivatives 4.0 International License. https://doi.org/10.1515/9783110661804-004 
souveraine que pour les élites nobiliaires, urbaines et ecclésiastiques de la Monarchie. Réunis, ces peintres forment un véritable réseau d'artistes employés dans et par les différents territoires de la Monarchie. Ce second chapitre prend en compte les portraits de Marie-Thérèse exécutés par des artistes de Vienne et surtout à Vienne. Après avoir envisagé dans le chapitre précédent la question du peintre de cour et du rôle de l'Académie, peut-on parler d'un portrait royal élaboré à Vienne principalement sur la demande de la cour viennoise ? Hormis la question des structures académiques et curiales, peut-on parler d'une influence viennoise ou non ? Force est en effet de constater qu'une bonne partie du corpus des portraits de Marie-Thérèse peut être considérée comme une " peinture viennoise », avec des portraits exécutés par des peintres autrichiens ou par des artistes qui sont basés à Vienne, en particulier autour de l'atelier de Martin van Meytens.

\section{Martin van Meytens, un peintre entre cour et Académie}

Jamais l'empire des artistes n'aurait resplendi avec un Apelle, un Léonard de Vinci, un Raphael, d'un Buonarotti et un Titien si la bienveillance et la protection d'un Alexandre de Macédoine, d'un roi François, d'un pape Léo, d'un Empereur Charles, n'avait insufflé à ces hommes encore plus de zèle pour leur art et ne les avait rendus encore plus grands ${ }^{100}$.

Telles sont les comparaisons évoquées par Scheyb dans sa lettre dédicatrice à Meytens au début de son ouvrage Natur und Kunst, Nature et art. Scheyb établit clairement un lien entre la gloire, la renommée et le succès de certains peintres et ceux de leurs commanditaires princiers, qu'ils soient conquérants, papes, princes ou empereurs.

Si beaucoup de peintres représentent Marie-Thérèse au cours de son règne, Martin van Meytens et son atelier réalisent sans aucun doute la plupart des portraits de la souveraine entre 1740 et 1770 . Entre la moitié et les trois-quarts des portraits lui sont attribués, ainsi qu'à son atelier et à ses élèves, sans évoquer les peintres de second rang qui s'inspirent et imitent ses représentations.

Les années comprises entre 1740 et 1760 constituent les grandes décennies d'activité et de triomphe de cet atelier comme de l'image, ou des images, de MarieThérèse. Meytens contribue à la création de l'image de Marie-Thérèse et réciproquement la famille impériale renforce la célébrité du peintre qui travaille aussi pour d'autres commanditaires que ceux issus du cercle de la famille impériale.

D’origine suédoise et flamande, le peintre Meytens arrive dès 1721 à la cour de Vienne où il travaille pour l'empereur Charles VI. Il réalise pour ce dernier de

100 Scheyb, Köremons, pp. 12-13. 
nombreux portraits et obtient par la suite la permission de travailler quelques années en Italie afin de parfaire ses connaissances et son style, en particulier d'améliorer sa technique de la peinture à l'huile ${ }^{101}$. Après un voyage de presque une décennie qui le conduit, entre autres, à Venise, Rome, Florence, Milan, Turin et Gênes, il revient en 1730 à Vienne où il reçoit immédiatement de nombreuses commandes des nobles ainsi que de la cour impériale ${ }^{102}$. Ceci est un point très important car si Martin van Meytens est le peintre de la cour impériale sous Charles VI et Marie-Thérèse, c'est aussi le peintre des nobles, des grands, des lettrés et des bourgeois de son époque. Les souverains sont en réalité des clients parmi d'autres.

Apprécié des grands de la Monarchie, Martin van Meytens l'est également des princes de son temps. Lors de son séjour à Paris à partir de 1717, le tsar Pierre le Grand avait tenté, sans succès, de le convaincre de venir travailler pour lui à la cour de Saint-Pétersbourg. Malgré le refus de l'artiste, le tsar aurait rapporté environ quarante portraits en miniature exécutés par Meytens ${ }^{103}$. Il ne resta pas le seul à rechercher les talents de celui-ci. Les rois de SardaignePiémont et de Suède, Auguste II de Saxe et plus tard la Grande Catherine de

101 Nagler, Künstler Lexikon, Neues Allgemeines Künstler-Lexikon, p. 237. Voir « La vie de Mr. Meytens à Vienne communiquée par lui-même. Fait à Vienne $1^{\text {er }}$ Juillet 1755 », Extrait de Briefe über die Kunst von und an Christian Ludwig von Hagedorn, Torkel Baden (Dir), Leipzig, 1797, cité par Lisholm, Martin van Meytens, pp. 129-131, ainsi que « Leben Herrn Martin von Meytens dessen Tod wir im 16ten Stück der Zeitung ausgezeigt haben », in Kunstzeitung der Kaiserlichen Akademie zu Augsburg, (1770), cité également par Lisholm, Martin van Meytens, pp. 115-124, p. 117. Ces deux textes, datés de 1755 et de 1770, dont le premier n'aurait probablement pas été rédigé par Martin van Meytens lui-même, sont les deux principaux écrits nous renseignant sur l'artiste et remontant au XVIII ${ }^{\mathrm{e}}$ siècle. S'il s'agit naturellement de conserver la prudence nécessaire sur ces documents, ils prouvent cependant, à leur manière, le succès, ou du moins la réputation, acquis par Meytens dès le XVIII ${ }^{\mathrm{e}}$ siècle, et même dès son vivant. Voir aussi Weissenhofer, "Martin de Meytens und der Wiener Hof », pp. 45-57, p. 49; Lisholm, Martin van Meytens, p. 133.

102 "Leben Herrn Martin van Meytens », in Kunstzeitung der Kaiserlichen Akademie zu Augsburg, (1770), in Lisholm, p. 120: "Sein Ruhm breitete sich nun immer mehr aus; und es war kein Hof in Europa, der nicht von ihm Arbeiten verlangt hätte. [. . .] Seine Figuren sind sehr zierlich und die Drapperien gross und edel. Seine Farbengebung ist der Naturgemäss und lebhaft. Kurz, ist es eine glückliche Zusammensetzung des Starken und Gewaltigen der Italiäner und des Reizenden und Anmuthigen der Franzosen. Sa gloire se répand de plus en plus; il n'y avait aucune cour en Europe qui n'ait pas réclamé des travaux de lui [. . .] ses personnages sont très gracieuses et les draperies grandes et nobles. Sa couleur est naturelle et vivante. Bref, c'est une réunion réussie de la force et de la grandeur des Italiens ainsi que du charme et de la grâce des français ".

103 Voir « La vie de Mr. Meytens à Vienne communiquée par lui-même. Fait à Vienne $1^{\text {er }}$ Juillet 1755 », Extrait de Briefe über die Kunst von und an Christian Ludwig von Hagedorn, Torkel Baden (Dir), Leipzig, 1797, cité par Lisholm, Martin van Meytens, pp. 129-131, p. 129. 
Russie auraient tous essayé en vain de l'attirer à leurs cours au détriment de Vienne. Voilà ce qu'on peut lire dans la revue artistique de l'Académie d'Augsbourg, à la mort de l'artiste en $1770^{104}$ : « Si c'est un bonheur de s'acquérir la faveur des grands, Meytens était l'un des hommes les plus heureux, car les monarques les plus remarquables qui régnaient alors en Europe cherchèrent à l'attirer à leur service ».

Une fois ses portraits terminés, les œuvres de Meytens suscitent un fort engouement et jouissent d'une grande popularité. Très enthousiaste, l'empereur Charles VI le fait nommer peintre impérial de chambre et de cour, kaiserlicher Hof-und Kammermaler, à la fin des années 1720. L'empereur et son épouse l'impératrice Élisabeth Christine sont proches du peintre, comme plus tard MarieThérèse, son époux et leur famille, qui rendent régulièrement visite au peintre pour le voir travailler ${ }^{105}$. Le portrait de Marie-Thérèse en robe de dentelle rose qui se trouve dans la salle de cérémonie du château de Schönbrunn (P 121) ${ }^{106}$, offre un certain nombre de détails intéressants. Par la qualité et la précision des détails, ce portrait est assez caractéristique de l'art de Martin van Meytens. Par exemple en arrière-plan du tableau, des objets comme l'équerre, la pyramide, l'œil de Dieu, sont représentés ${ }^{107}$.

Les portraits réalisés par Meytens sont en règle générale des portraits à l'huile, de grandes dimensions. La souveraine y est peinte en pied, de trois

104 « Leben Herrn Martin von Meytens, dessen Tod wir im 16ten Stück der Zeitung angezeigt haben ", in Kunstzeitung der Kaiserlichen Akademie zu Augsburg, (1770), cité d'après Lisholm, Martin van Meytens, p. 117 : « Wenn es ein Glück ist, die Kunst [sic] der Grossen sich zu erwerben : so war Meytens einer der glücklichsten Menschen ; denn die merkwürdigsten Monarchen, die damals in Europa waren, suchten ihn in ihre Dienste zu ziehen ».

105 Lisholm, Martin van Meytens, p. 130 ; Weissenhofer, « Martin de Meytens », p. 50. En plus de ses visites personnelles, la souveraine aurait envoyé ses propres enfants chez Meytens. En dehors de ses activités artistiques, Meytens s'intéresse particulièrement à la physique, à la chimie ainsi qu'à l'alchimie. Il obtient de Marie-Thérèse le privilège de monter une fabrique de couleurs, comme en témoignent les couleurs très vives que l'on retrouve sur les robes de MarieThérèse dans ses portraits ; voir Baum, Katalog des Österreichischen Barockmuseums, vol. 2, p. 425 ; Lechner, « Martin van Meytens », p. 14 ; Lisholm, Martin van Meytens, pp. 122, 131.

106 Le portrait de Marie-Thérèse en robe de dentelles rose fait partie de la commission de Primogeniturfideikommisses, c'est-à-dire qu'elle est au cœur des collections des Habsbourg. Ce tableau porte le numéro 8762 dans la galerie de tableaux du Kunsthistorisches Museum - ce qui signifie que le portrait ne fut inventorié que tardivement. Le tableau est toutefois déjà mentionné et reproduit dans l'Österreichischen Kunsttopographie (2 volumes, Die Denkmale der Stadt Wien, XI.-XXI. Bezirk), dès 1908 et ce dans la salle des cérémonies.

107 On peut éventuellement y lire un rappel de l'activité maçonnique de Meytens. Sur l'histoire de la franc-maçonnerie en Autriche au XVIII ${ }^{\mathrm{e}}$ siècle, voir Kodek, Von der Alchemie zur Aufklärung ; Krivanec, « Die Freimaurerei »; Reinalter, Aufklärung, Humanität und Toleranz. 
quarts, le regard légèrement tourné vers le spectateur. Marie-Thérèse apparaît alors de manière particulièrement majestueuse et imposante. La manière de peindre de Meytens est minutieuse, jusqu'au moindre détail, en particulier pour la reproduction des tissus et des couleurs. Outre la peinture italienne, l'art de Meytens est influencé par la peinture hollandaise de l'époque moderne. Celle-ci se caractérise particulièrement par l'attention portée aux tissus et aux détails très finement représentés. Le goût de Meytens pour les étoffes est en effet remarquable. Il est très influencé par le style du portraitiste de la cour d'Angleterre d'origine flamande, Anton van Dyck ${ }^{108}$. Toutefois, ses séjours en Italie, très courants à l'époque moderne, jouent également un rôle important dans la formation de son style et surtout de sa manière de peindre ${ }^{109}$. Dans les portraits, les habits de Marie-Thérèse sont soignés et luxueux, les couleurs illuminent le personnage harmonieusement ${ }^{110}$.

Martin van Meytens contribue à la formation d'une image majestueuse de Marie-Thérèse. Il s’appuie sur différents héritages italiens et flamands. Meytens est aussi marqué par le classicisme français, en particulier par le peintre français Hyacinthe Rigaud. Outre le célèbre portrait de Louis XIV, Rigaud a exécuté divers portraits pour les membres de l'aristocratie autrichienne comme par exemple pour Philipp Ludwig von Sinzendorf ${ }^{111}$.

L'art de peindre et le style de Meytens satisfont le goût de l'époque, tel que la cour le prise ${ }^{112}$. Les grandes dimensions des tableaux, les vêtements peints avec un grand sens de la précision, l'attention particulière apportée aux couleurs et aux détails, ravissent les grands. Malgré ces diverses influences, le style pictural de Meytens possède sa propre originalité ${ }^{113}$. Marie-Thérèse lui donne de plus en plus de preuves de sa confiance et de sa reconnaissance en lui rendant régu-

108 Lechner, «Martin van Meytens », p. 11.

109 Sur le séjour italien, voir Lechner, « Martin van Meytens », pp. 12-13 ; Lisholm, Martin van Meytens, pp. 22-27, 117-119, 125-126, 129-130 ; Weissenhofer, « Martin de Meytens », pp. 48-49.

110 Baum, Katalog des Österreichischen Barockmuseums, vol. 2, p. 425.

111 Schreiden, « Jacques van Schuppen », p. 61.

112 Voir « La vie de Mr. de Meytens à Vienne communiqué par lui-même. Fait à Vienne 1 Juillet 1755 », Briefe über die Kunst von und an Christian Ludwig von Hagedorn, Torkel Baden (Dir), Leipzig, 1797, cité par Lisholm, Martin van Meytens, pp. 129-131; “ Leben Herrn Martin von Meytens, dessen Tod wir im 16ten Stück der Zeitung angezeigt haben », in Kunstzeitung der Kaiserlichen Akademie zu Augsburg, (1770), cité d'après Lisholm, Martin van Meytens, p. 119 : « Kaum hatte man die erste Arbeit von ihm gesehen ; so wollte schon jedermann von diesem Meister gemalet seyn ; einige in Schmelzwerk, andere in Miniatur, und noch andere auf grosse Manier [. . . ]. Es war ihm nicht sehr angenehm, dass er in so kurzer Zeit mit Arbeiten überhäuft wurde ».

113 Heinz, « Bemerkungen », pp. 280-281. 
lièrement visite dans son atelier en compagnie de son époux ${ }^{114}$. Lorsque le temps lui manque, elle envoie au peintre des esquisses. Toutefois, une certaine rivalité semble s'être installée entre Meytens et un autre peintre très proche de MarieThérèse, le Genevois Jean-Étienne Liotard, la souveraine posant apparemment plus volontiers pour Liotard que pour Meytens ${ }^{115}$. Selon nous cependant, il ne peut pas s'agir d'une véritable concurrence car les portraits de Liotard n'obéissent pas nécessairement aux mêmes objectifs que ceux de Meytens. Ils sont destinés à un usage plus familier et personnel. C'est peut-être la raison pour laquelle MarieThérèse aurait pu préférer poser pour Liotard davantage que pour Meytens.

Comme peintre de cour, Meytens est très proche de l'entourage impérial. Il peut ainsi être considéré comme un familiaris de la famille impériale. Meytens se sert en général d'un modèle de tête et de corps pour reproduire les portraits de Marie-Thérèse. Certains éléments de ses portraits reviennent régulièrement comme la coupe de cheveux, la pose, le cou, les bijoux ou encore les gestes des mains. À l'instar des membres de son atelier, Meytens utilise les mêmes études pour exécuter diverses répliques des portraits de Marie-Thérèse et des siens ${ }^{116}$.

L'organisation de l'atelier de Martin van Meytens n'est pas sans faire songer à une véritable « industrie » dans la mesure où les tâches sont partagées et réparties entre les différents artistes de l'atelier, chacun selon ses spécialités, ses talents et ses compétences. Ce mode de travail est habituel à cette époque, bien que l'atelier de Meytens soit plus grand et complexe que d'autres. Les portraits originaux sont, en règle générale, conservés à Vienne tandis que les répliques sont dispersées dans les pays héréditaires et dans l'espace allemand du Saint Empire ainsi que dans toute l'Europe. Ces dernières sont reproduites en grande quantité, avec à peu près la même qualité, par l'atelier de Martin van Meytens. Attribuer avec certitude un peintre pour chaque œuvre se révèle donc en bien des cas une entreprise difficile. Les membres de l'atelier de Meytens se spécialisent dans la réalisation d'une partie du portrait. Les artistes, assistants et élèves, effectuent ainsi différentes tâches ${ }^{117}$. Ce travail en atelier, en série, renvoie au travail de montage que l'on retrouve quelques siècles plus tard au début de la photographie. Le neveu et élève de Meytens, Sophonias de Derichs originaire de Stockholm, reproduit tout particulièrement les accessoires tandis

114 « Leben Herrn Martin von Meytens, dessen Tod wir im 16ten Stück der Zeitung angezeigt haben », in Kunstzeitung der Kaiserlichen Akademie zu Augsburg, (1770), cité d'après Lisholm, Martin van Meytens, p. 120.

115 Koschatzky, « Jean-Etienne Liotard », pp. 309-310, 315-316. Merci à Elfriede Iby d'avoir attiré notre attention sur ce point.

116 Weissenhofer, « Martin de Meytens », p. 50.

117 Baum, Katalog des Österreichischen Barockmuseums, vol. 2, p. 425. 
que c'est un Français, dont on ignore aujourd'hui le nom, qui s'occupe de la représentation des dentelles ${ }^{118}$. C'est un élément particulièrement important car les dentelles renvoient à l'activité manufacturière des Pays-Bas autrichiens qu'il s'agit de mettre en valeur. Un certain J. G. Canton réalise les scènes de bataille en arrière-plan. Pour les portraits de groupes et d'évènements, des dénommés Dalling, Fischer, Greipel, Pohl, Rebell, Sigrist et Schinnagl participent à la réalisation des tâches ${ }^{119}$.

Les artistes d'ateliers, formés en tant qu'artisans, s'inscrivent en grande partie dans la tradition italienne de peinture. À la suite de l'activité de Meytens pour l'Académie dès 1759 , le cercle des élèves de son atelier et de ses collaborateurs s'élargit considérablement. Certains peintres employés dans l'atelier de Meytens comme l'artiste Johann Joseph Dollenstein ${ }^{120}$, peintre d'origine tchèque, réalisent également, à la demande de hauts dignitaires, des portraits de Marie-Thérèse. Le président de la Chambre de Cour Karl Ferdinand Koenigsegg, ou Königsegg, de Rothenfels commande à Vienne en 1751 auprès de Dollenstein, qui travaille au demeurant à Vienne, des portraits du couple impérial ainsi que son propre portrait (P 54) ${ }^{121}$.

Malgré le grand nombre d'élèves de Meytens, la plupart des œuvres lui sont principalement attribuées à lui, le maître. C'est alors habituel. Beaucoup d'autres artistes s'inspirent de sa manière de peindre tout au long du règne de Marie-Thérèse, la notion d'originalité des œuvres d'art n'ayant alors pas lieu d'être. Martin van Meytens marque l'imagination des générations suivantes en proposant des images standardisées de Marie-Thérèse qui ornent les livres scolaires des anciens pays de la Monarchie jusqu'à nos jours, en particulier les portraits conservés au château de Schönbrunn et à l'Académie des Beaux-Arts de Vienne. De même, les portraits de famille sur la terrasse du château de Schönbrunn (P 140, P 141, P 142, P 144, P 145) sont aujourd'hui encore recopiés dans les dictionnaires, les catalogues d'exposition et dans les autres ouvrages sur la Monarchie destinés au grand public.

118 Toutefois, selon Georg Lechner, c'est à De Derichs qu'aurait été confiée la mission de peindre les dentelles et les robes ; voir Lechner, « Martin van Meytens », p. 18.

119 Baum, Katalog des Österreichischen Barockmuseums, vol. 2, p. 425. Concernant les élèves de Martin van Meytens, voir aussi Heinz, « Bemerkungen », p. 282; Lechner, « Martin van Meytens », pp. 16-20.

120 Ce peintre n'est pas recensé dans les lexiques biographiques d'artistes. Toutefois le Musée Josef Kollar de Banská Štiavnica nous renseigne qu'il s'agit d'un artiste d'origine tchèque.

121 Čelková, « Die Bergbaukunst », p. 46; Čelková, « Der Kaiser kommt », p. 253; Čelková, «Imperial visits of the Habsburgs ", p. 55. 
L'époque viennoise de Martin van Meytens peut se répartir en deux périodes : l'une où il n'est « que » peintre de cour (1729-1759) et l'autre où il assume, en plus de son activité de peintre de cour, la fonction de directeur de l'Académie (1759-1770). L'atelier de Meytens semble alors pleinement fonctionner comme une entreprise plus ou moins liée à l'Académie qu'il dirige. Il s'entoure d'élèves pour son propre atelier ${ }^{122}$.

Durant les dernières années du peintre, alors que Marie-Thérèse est déjà veuve, le principal et le plus monumental portrait peint par Meytens un peu avant sa mort est réalisé vers 1765: il se trouve actuellement au Palais Trautson à Vienne $(\mathrm{P} 180)^{123}$.

En raison d'une demande croissante de la cour, Martin van Meytens devient un portraitiste très prisé et son art du portrait suscite un vif engouement. Le succès de l'artiste n'est pas lié uniquement à un phénomène de mode mais répond aussi à certaines attentes de ses contemporains qui souhaitent se voir à la manière des rois et des nobles français et italiens.

Les autres grandes familles du pays, engagés avec la Maison impériale dans la construction de l'ensemble étatique, partagent le goût de celle-ci en matière de représentation picturale. Les Liechtenstein, les Harrach, Pálffy, Kuefstein, Schwarzenberg, Daun, Tarouca, Khevenhüller, Cobenzl comptent parmi les clients de Meytens ${ }^{124}$. Un esprit d'émulation anime les rapports entre les nobles et les souverains, chacun désirant posséder des portraits, les siens ou ceux de la famille impériale, des mêmes artistes.

Dans sa propre maison, à Vienne, non loin du château de Schönbrunn, Meytens reçoit un certain nombre de personnalités royales, diplomatiques et ecclésiastiques pour lesquelles il exécute des portraits. La proximité de Schönbrunn permet à l'artiste de rester toujours proche de la cour et de sa souveraine.

Divers circuits organisent et structurent la commande auprès des artistes, et notamment auprès de Meytens. Certaines commandes de la cour sont attestées dans les comptes du Geheimes Kammerzahlamt, comme par exemple une paire de portraits de Marie-Thérèse et de François Étienne envoyés en 1745, lorsou en prévision du couronnement impérial de ce dernier, à l'archevêque-électeur de Cologne. Meytens reçoit quarante ducats pour chacun des tableaux ${ }^{125}$. On trouve encore quelques bribes de la correspondance du peintre avec ses commanditaires et clients, indiquant la popularité de ses portraits de Marie-

122 Lechner, « Martin van Meytens », pp. 16-20.

123 Lisholm, Martin van Meytens, p. 100.

124 Lisholm, Martin van Meytens, p. 52 et p. 59; Weissenhofer, « Martin de Meytens », p. 51.

125 HHSTA HA GehKZ 1-19, Karton 1, HZA Nr. 19, f 1-161, f 16. 
Thérèse auprès de différents types de publics. Dans une lettre destinée à Karl comte Cobenzl, le 22 juin 1743, Meytens répondait à une missive de celui-ci du 12 février de la même année :

J'ay cru qu'il serait mieux d'attendre à y faire réponse jusqu'à ce que le portrait [que Votre Excellence] m'a demandé de la Reine fut prete. Je suis bien mortifié que cela ait tant tardé, mais j'espère qu'Elle me pardonnera, ayant été empeché par les ouvrages indispensables qu'il m'a fallu faire pour la Reine. Je m'estimeray très heureux si le portrait que je vay vous envoyer par le moyen de Grunerischer Wechselstuben, vous donne toute la satisfaction que je souhaitais, n'ambitionant rien que de mériter l'honneur de votre approbation et la continuation de vos bonnes graces.

Et il ne manquait pas de rappeler à son commanditaire qu'il attendait un paiement rapide :

Comme je viens d'apprendre de Messieurs Grüner qu'ils n'ont point d'ordre de me payer, j'espère que Votre Excellence ne tardera pas de le faire, afin que cela ne retarde pas l'expédition du portrait: Elle sait déjà que ce sont Trente Ducats d'or qui me reviennent ${ }^{126}$.

Cette lettre témoigne de la fréquence des commandes passées auprès du peintre, mais aussi du fait que les commanditaires payaient parfois de manière irrégulière. Cela peut être aussi le cas de Marie-Thérèse, encore plus souvent à court d'argent que les autres nobles. Le portrait du souverain s'inscrit donc dans le registre des portraits aristocratiques du temps. Les nobles imitent les portraits des souverains même si l'émulation reste réciproque. Le souverain est en effet le " premier » parmi les membres de son aristocratie qu'il a souvent contribuée lui-même à former.

D'autres sources encore attestent des travaux de Meytens pour les grandes familles de la Monarchie. Des comptes dans les archives Schwarzenberg mentionnent une dépense de 20 ducats ou 82 florins et demi en 1743, pour un portrait de la reine Marie-Thérèse ( $P$ 243). L'année suivante, Meytens reçoit 100 ducats pour un portrait de groupe de quatre enfants du couple royal, assis sur un canapé jaune. Ces portraits royaux sont commandés en parallèle, semble-til, avec des portraits de famille des Schwarzenberg, dont un de la princesse Maria Theresia née Liechtenstein, épouse du prince Joseph Adam Schwarzenberg, et un portrait de leurs trois enfants ${ }^{127}$. Détail intéressant à noter, le portrait de la souveraine revient moins cher que celui de la princesse, qui coûte 25 ducats. En 1748, une facture écrite par Meytens évoque encore quatre tableaux

126 OeStA-HHStA, Große Korrespondenz 265-21, Meytens à Karl Graf Cobenzl 1743, f 6 631, 632, 633; voir aussi Lisholm, Martin van Meytens, p. 56.

127 Mörath, "Martin von Meytens », pp. 1-2; voir aussi Lisholm, Martin van Meytens, pp. $55-56$ et 59 . 
exécutés pour les Schwarzenberg, deux paires de portraits du prince et de la princesse, représentés une fois à cheval et une fois assis, dont le prix total s'élève à 272 ducats $^{128}$. Une grande ressemblance se lit à travers toutes ces commandes de tableaux: il existe une véritable imitation réciproque.

Dans les inventaires de la famille Harrach, les peintres de cour, Meytens, ainsi que Kobler et Hickel, sont cités ${ }^{129}$. Les Harrach constituent un bon exemple pour illustrer la participation des commanditaires à l'élaboration des portraits royaux. Comme les Schwarzenberg, les membres de la famille Harrach ont pu commander leurs portraits et ceux de leur famille, ainsi que ceux de la famille impériale, auprès des peintres de cour, en premier lieu auprès de Meytens. Friedrich August Harrach a ainsi exprimé le souhait d'avoir un portrait de la souveraine en habit de couronnement avec la couronne sur la tête. Son frère le comte Bonaventura annonce, quant à lui, le 10 octobre 1742 un léger changement dans la composition par rapport à son arrangement usuel. Dans la correspondance de la famille Harrach, nous pouvons lire : « Je vous ferai venir le portrait de la reine habillée comme elle l'a été le jour du couronnement ${ }^{130}$ avec la seule différence ce que la couronne ne faisant pas bon effet en peinture sur la tête, elle est placée à côté ${ }^{131}$. Les commanditaires de portraits, en particulier nobiliaires, participent ainsi à la représentation de leur souveraine.

Martin van Meytens influence et inspire d'autres artistes de renom dans leur propre représentation de Marie-Thérèse. Cette influence ne se limite pas seulement au domaine du portrait. Les deux statues réalisées par le sculpteur Franz Xaver Messerschmidt semblent s'inscrire très clairement dans la lignée de Meytens. Élève de l'Académie, Messerschmidt exécute un certain nombre de commandes officielles entre 1760 et 1763 . La commande pour la statue de l'empereur n'a lieu qu'après le décès du souverain en 1765. Martin van Meytens aurait probablement joué un rôle décisif dans la décision de Marie-Thérèse de passer commande auprès de Messerschmidt. Il s'agit de portraits officiels sculptés où les souverains portent des couronnes sur la tête et tiennent les insignes

128 Mörath, « Martin von Meytens », pp. 2-3.

129 OeStA-AVA, FA Harrach Fam. in spec. 528.1, 529.1. Harrach, Graf Ferdinand Bonaventura II, Bruder, Korrespondenz mit Friedrich August Harrach, 1741 et 1742-45; AVA FA Harrach Fam in spec 775; Gräflich Harrach'sches Familienarchiv; Kunst und Wissenschaft; Hauptverzeichnis der Erlauchtgräflich Harrachschen Bilder Gallerie; Schloss Bruck.

130 Il s'agit du couronnement hongrois en considération de la date.

131 AVA FA Harrach Fam. in specie, Nr. 529 Korrespondenz Friedrich August, lettre du 10 octobre 1742, citée aussi par Lisholm, Martin van Meytens, pp. 56-57, p. 56 ; Weissenhofer, « Martin de Meytens », p. 51. 
dans la main. Marie-Thérèse est représentée en tant que souveraine légitime et établie des territoires héréditaires tandis que François Étienne est représenté à la tête du Saint Empire romain germanique ${ }^{132}$.

Meytens et son atelier ne sont pas les seuls peintres de cour et d'Académie à représenter Marie-Thérèse. Durant les quatre décennies du règne, d'autres peintres très productifs à la cour de Vienne, participent également à l'élaboration de l'image royale, même si leur nom n'est pas resté aussi connu pour la postérité.

\section{Divers peintres très actifs à et pour la cour de Vienne}

Le premier peintre connu à avoir représenté la jeune princesse est le Danois Andreas Möller (1684-1762) qui peint, sur ordre de Charles VI, un portrait de Marie-Thérèse jeune fille ( $\mathrm{P} 1$, Figure 1). Ce portraitiste et peintre de miniatures travaille pour de nombreuses cours européennes. Entre 1715 et 1721, il est peintre de cour auprès de Charles de Hesse-Kassel, avant de travailler à la cour de Vienne pour l'empereur ${ }^{133}$. C'est vers 1727 que Möller réalise son portrait de Marie-Thérèse $^{134}$. Représentée avec des fleurs sur le bas de la robe et dans les mains, la jeune archiduchesse pose délicatement à côté du chapeau archiducal placé sur un coussin. Les portraits des filles de l'empereur Joseph $\mathrm{I}^{\mathrm{er}}$, les archiduchesses Marie-Josèphe et Marie-Amélie, par Johann Kupetzky ont probablement servi de modèle. Une gravure de Joseph Schmuzer, réalisée d'après ce portrait, porte l'inscription « Andreas Möller pinxit Viennae 1726 », raison pour laquelle le portrait de Marie-Thérèse est attribué à l'artiste danois ${ }^{135}$.

132 Pötzl-Malikova, « Die Statuen Maria Theresias und Franz I. Stephans », pp. 132-133, p. 136.

133 Holck Colding, "Møller »; Repetzky, « Møller »; Schidlof, La miniature en Europe, vol. 2, p. 581; Učníková, Portréty Márie Terézie, p. 59.

134 Le portrait est au Kunsthistorisches Museum de Vienne mais était autrefois exposé au château d'Hetzendorf en Autriche.

135 Krasa-Florian, « Jugendbildnis Maria Theresias »; voir aussi Iby, Telesko, « Hofmobiliendepot », p. 263; Iby et al., « Maria Theresia », p. 29; Kuster, « Erzherzogin Maria Theresia ». Un second exemplaire de ce portrait de Marie-Thérèse se trouve dans la Gemäldegalerie de Dresde. 


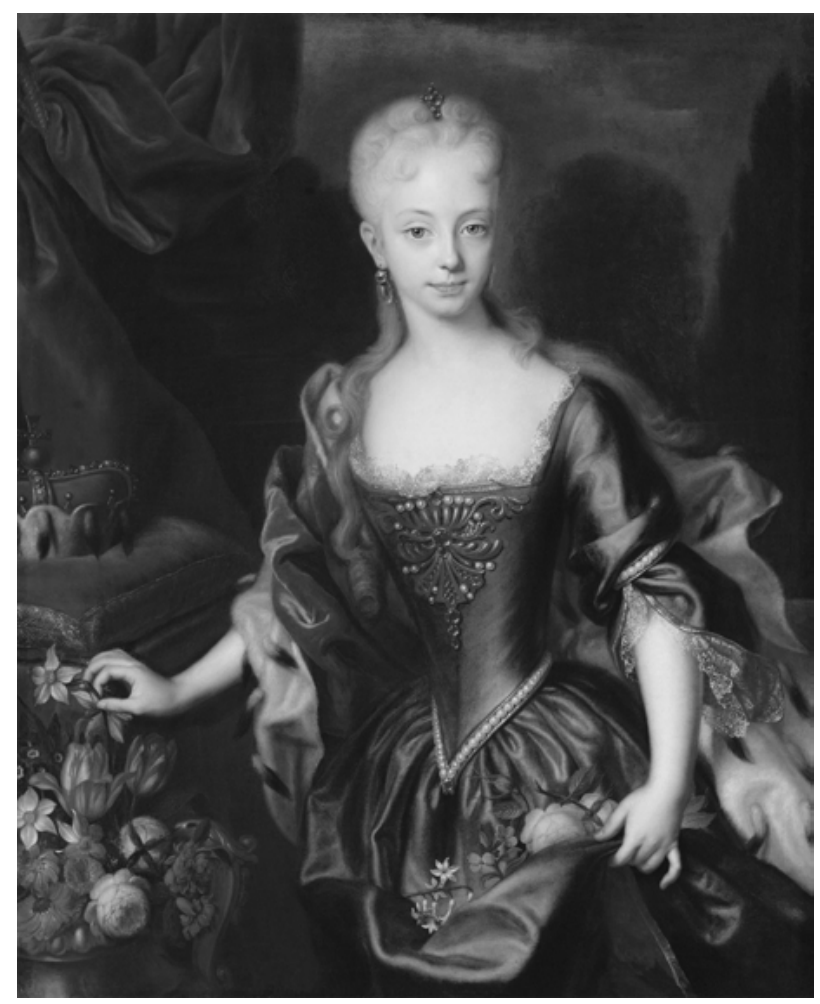

Figure 1: Andreas Möller, Marie-Thérèse à l'âge de onze ans, vers 1727, 94 x $75 \mathrm{~cm}$, Ambras, Kunsthistorisches Museum Vienne, Gemäldegalerie, Inv. Nr. GG 2115, Autriche, (c) « KHM-Museumsverband ».

Si les portraits s'échangent entre les cours princières, ce mouvement d'échange touche également les peintres qui, comme Andreas Möller, sont actifs à divers endroits. À côté de ces artistes itinérants, des familles et des dynasties de peintres se mettent en place tout au long de l'époque moderne. C'est en particulier le cas des Hamilton tout comme des Auerbach ou des Palko.

Durant les premières années du règne, dans les années 1740, Philipp Ferdinand von Hamilton (1664-1750), fils du peintre James Hamilton, est un artiste familier de la dynastie. À l'instar de plusieurs autres qui exécutent des portraits de Marie-Thérèse, Hamilton est originaire de Bruxelles. Il s'installe par la suite à Vienne. Sa présence y est recensée dès 1705 comme peintre impérial de chambre, Kaiserlicher Kammermaler. À Vienne, il travaille en effet successivement pour Joseph $\mathrm{I}^{\mathrm{er}}$, pour Charles VI, puis pour Marie-Thérèse. Hamilton réalise des portraits de ces trois souverains. Comme beaucoup de grands peintres de son époque, tel 
Meytens, Philipp Ferdinand von Hamilton a également peint les portraits des membres de la noblesse autrichienne. On retrouve ainsi beaucoup de ses œuvres dans les châteaux et dans des collections privées. Il signe fréquemment ses tableaux de cette manière : « Philip. F. de Hamilton S[acrae] C[aesareae] M[aiestatis] $C$ [urialis] $\mathrm{P}$ [ictor] ${ }^{136}$. L'artiste réalise en outre des peintures équestres, comme pour le gendre de Marie-Thérèse, Albert de Saxe-Teschen, époux de la fille préférée de la souveraine, Marie-Christine. Ce n'est donc pas un hasard que ce soit Hamilton qui, avec le peintre officiel Martin van Meytens, ait exécuté des portraits de Marie-Thérèse à cheval pour célébrer la cérémonie du couronnement hongrois à Presbourg (P 18, P 19). La peinture équestre correspond d'ailleurs à une tradition picturale flamande bien établie, en particulier au sein de la famille d'artistes des Hamilton, puisque son frère Johann Georg Hamilton a également exécuté un certain nombre de représentations équestres pour la cour ${ }^{137}$.

Outre des portraits, le peintre de cour Hamilton continue toutefois d'exécuter des natures mortes et des tableaux d'animaux, genres inférieurs à la peinture d'histoire. Ce détail est révélateur de la diversité des œuvres que sont alors amenés à réaliser les peintres. Hamilton apparaît comme un salarié de la cour de Vienne avec une rémunération relativement fixe et des missions à accomplir. Les registres de l'Office de comptes de la Chambre, les Kammerzahlamtsbücher, le recensent comme un Camer Mahler ${ }^{138}$ chargé de livrer chaque année deux peintures en échange de la somme de cinq cents florins. Certains artistes de cour sont donc payés régulièrement et peuvent même prétendre à une augmentation comme en témoigne le cas de ce peintre. Arrêtons-nous sur des extraits de registres d'art issus des Hofparteienprotokollen, des protocoles de cour, de l'Obersthofsmeisteramt, du grand maître de cour, qui s'étendent entre 1637 et $1780^{139}$. Les protocoles du grand maître de cour se trouvent dans les archives de cour et les fonds privés et familiaux, Hofarchive, Privat- und Familienfonde ${ }^{140}$. Le peintre Hamilton ${ }^{141}$ y ap-

\footnotetext{
136 Kugler, « Hamilton, Philipp Ferdinand ».

137 Kugler, « Hamilton, Johann Georg ».

138 Schatzmeister Gallerie Inspector Adjunct Camer Mahler Hn Philipp Ferdinand von Hamilton, années 1740, FHKA SUS KZAB Kammerzal Zahlamtsbücher. Bancaliteats Cameral Zahlamts Rechnung Anno 1742, BD. 29, fo. 52. Hamilton est également mentionné dans les livres Cameral Zahls Amtsrechnung 27 de l'année 1740, 30 de l'année 1743, 31 de l'année 1744 et le 32 de l'année 1745.

139 Pillich, « Kunstregesten aus den Hofparteienprotokollen des Obersthofmeisteramtes von 1638-1780 », vol. 17/18, pp. 639-645; 1741, p. 641; 1742, 1743, p. 642, 1747, p. 643.

140 Pillich, 17 Hofparteienprotokoll 1741-1744; 19. Hofparteienprotokoll 1747-1748.

141 Pillich, 17 Hofparteienprotokoll 1741-1744; pp. 641-642; 472.29 Juin 1741 Presbourg, 478. 3 Juillet 1742 Vienne; 482. 25 Octobre 1742 Vienne; 485 18. Mars 1743 Vienne; 19. Hofparteienprotokoll 1747-1748, p. 643, 489. 19 Juin 1747 Vienne.
} 
paraît comme une sorte de fonctionnaire chargé de livrer régulièrement des portraits. Il n'est pas précisé de quels types de portraits il s'agit exactement.

Parmi tous les portraits mentionnés d'Hamilton, on y trouve ceux de MarieThérèse, en particulier deux portraits équestres de la souveraine et de son époux François Etienne sur la colline du couronnement de Presbourg, réalisés en $1742^{142}$ par Hamilton ( $\mathrm{P}$ 18) et qui semblent faire écho aux portraits similaires réalisés vers la même période par Meytens (P 19). Puis dès 1747, toujours en sa qualité d'artiste de cour, le peintre Hamilton continue de travailler de manière régulière pour celle-ci puisqu'il est censé livrer chaque année quatre tableaux à l'huile et recevoir en échange, chaque année, mille florins. Ce traitement lui est accordé, à sa demande, et surtout en raison de son grand âge. Quelques années auparavant, il ne devait livrer que deux tableaux par an. Désormais, il se doit d'en livrer quatre ${ }^{143}$. La production de portraits princiers augmente, répondant à une demande accrue de la part de la cour.

Si une bonne partie des portraits du corpus sont réalisés par Martin van Meytens et son atelier, une autre a été exécutée par d'autres peintres, également de cour et souvent académiciens, au premier rang desquels figure Peter Kobler. Durant les deux premières décennies du règne de Marie-Thérèse, Kobler est un artiste viennois très productif. Dans les années 1750, il est souvent cité dans les livres de comptes de la cour. En 1750, des portraits de Marie-Thérèse et de François Étienne sont exécutés par Kobler et envoyés à Milan ${ }^{144}$. En 1751, le peintre réalise de nouveau des portraits du couple impérial, cette fois pour

142 Pillich, 17 Hofparteienprotokoll 1741-1744, vol. 17/18, p. 641, 472. 29 Juin 1741 Presbourg ; en 1741, Hamilton a déjà livré un tableau, « à la grande satisfaction de la souveraine », pour lequel il perçoit 250 Gulden, la somme de virement est datée du 29 Juin 1741 de Presbourg, Marie-Thérèse est couronnée roi de Hongrie le 25 Juin 1741, il est aussi mentionné que Philipp Ferdinand Hamilton est Kammermaler depuis le 18 Juin 1741 ; Pillich, 17 Hofparteienprotokoll 1741-174, vol. 17/18, p. 642, 478. 3 juillet 1742 Vienne ; en juillet 1742 Philipp Ferdinand Hamilton reçoit 250 Gulden pour des tableaux déjà livrés en 1741, en octobre 1742, il gagne encore 250 Gulden après avoir livré un tableau, 482. 25 Octobre 1742 Vienne, et en mars 1743, 250 Gulden lui sont de nouveau donnés après avoir terminé un second tableau, 485 18. Mars 1743 Vienne.

143 Dans Pillich, 17 Hofparteienprotokoll 1741-1744, vol. 17/18, p. 641, 472. 29 Juin 1741 Presbourg ; il est mentionné qu'Hamilton doit dès 1741 livrer deux tableaux par an pour 500 Gulden, d'autres tableaux seront payés de manière séparée. Il a déjà rendu un tableau à la satisfaction de la souveraine. Pillich, 19 Hofparteienprotokoll 1747-1748, vol. 17/18, p. 643 ; 489. 19 Juin 1747 Vienne ; en 1747, il reçoit chaque année 1000 Gulden pour 4 huiles, en raison de son grand âge la souveraine lui accorde cette somme. Après la mort de l'artiste, sa veuve Marie-Thérèse Hamilton reçoit, à partir du $1^{\text {er }}$ janvier 1753, deux ducats par mois ; Pillich, 22 Hofparteienprotokoll 1753-1754, vol. 17/18, p. 650, 532. 8 Janvier 1753 Vienne.

144 Fleischer, Das kunstgeschichtliche Material, p. 57. 
Presbourg $^{145}$. S'agit-il des mêmes portraits que ceux que nous trouvons aujourd'hui dans ces villes ? Il se peut que le portrait de Marie-Thérèse, aujourd'hui au Musée historique de la ville de Bratislava, attribué à Peter Kobler et daté d'aprés 1745 (P 92) ${ }^{146}$, corresponde à celui mentionné dans les Kammerzahlamtsbücher vers 1751, à moins que ce dernier soit à identifier avec un tableau de Marie-Thérèse en roi de Hongrie exposé aujourd'hui au Hofmobiliendepot de Vienne. En 1752, Kobler réalise encore deux portraits du couple impérial, en pied, commandés pour récompenser le secrétaire d'État Johann Christoph baron de Bartenstein ${ }^{147}$. Enfin, mentionnons les portraits du couple impérial datés de 1759 et conservés dans la résidence d'Augsbourg qui sont attribués à Kobler (P 105) ${ }^{148}$.

Dès 1747, Kobler est nommé par Marie-Thérèse Kaiserlich-Königlicher Kammermaler, peintre de chambre impérial et royal. La souveraine finit même par l'anoblir en 1760. S’il peut apparaître comme le prototype même du peintre de cour, son nom n'est peut-être pas aussi lié aujourd'hui au portrait de MarieThérèse que peut l'être celui de Martin van Meytens. Toutefois, Kobler n'en produit pas moins plusieurs portraits de la souveraine ${ }^{149}$. Comme la plupart des peintres de cour, il travaille également pour d'autres commanditaires comme l'atteste l'exemple de l'abbaye de Sankt Florian (P 135, Figure 32), il n'est donc pas seulement tributaire de Vienne et de la commande impériale ${ }^{150}$.

\section{Johann Gottfried et Johann Karl Auerbach : une famille de peintres}

À la même époque, de véritables dynasties et familles de peintres travaillent pour les souverains, à l'exemple des Auerbach, père et fils, qui se mettent au service de Charles VI puis de Marie-Thérèse. Devenir peintre de père en fils est un fait courant à l'époque. Le père Johann Gottfried Auerbach (1687-1743) séjourne dès 1716 à Vienne où il devient en 1735 peintre de cour, Hofmaler, et en 1750 membre de l'Académie des Beaux-Arts. En 1741 sous Marie-Thérèse, on le retrouve sous le titre de Kammermaler. Dès 1728, il s'est déjà fait un nom auprès

145 Fleischer, Das kunstgeschichtliche Material, p. 60: «1751-86-besagten Cammer Mahler Kobler wegen 2 von Beeden K. K. Maytt. Allerhöchst Personen in Lebensgrösse verferttigt und gelieferten Portrait, so nacher Pressburg ist abgesendet worden ».

146 Marie-Thérèse y est représentée avec un sceptre et une seule couronne, la couronne impériale familiale des Habsbourg.

147 Fleischer, Das kunstgeschichtliche Material, p. 64.

148 Von Hagen, « Ensembles und Einzeldenkmäler », p. 170. Merci aussi au Dr. Christoph Nicht.

149 Haupt, Das Hof- und hofbefreite Handwerk, p. 321; Lechner, « Porträtmalerei », pp. 47, 50. 150 Korth et al., « Die Kaiserzimmer », p. 275; Kovács, « Maria Theresia und Franz I. Stephan ». 
de la cour où il exécute un portrait de Charles VI, aujourd'hui au Kunsthistorisches Museum de Vienne ${ }^{151}$. Son fils, Johann Karl Auerbach (1723-1788), est également un peintre d'histoire, en plus d'être portraitiste. Johann Karl suit un apprentissage auprès de son père et devient comme lui membre de l'Académie ${ }^{152}$. Avec d'autres artistes de son entourage, il réalise un portrait de Marie-Thérèse en habit de couronnement de Bohême daté de 1772, et destiné au château de Prague (P 76, Figure 31). Dans les archives de cour répertoriées par Julius Fleischer, ce tableau est recensé parmi un groupe de portraits de la famille impériale, attribués à Auerbach et livrés pour la résidence impériale de la capitale de Bohême ${ }^{153}$.

L'abbaye bénédictine de Břevnov près de Prague conserve dans sa salle de réception un portrait de Marie-Thérèse avec l'archiduc Joseph enfant, réalisé en 1743 (P 77). Ce portrait a certainement été exécuté par Auerbach père, ou du moins il lui est attribué. Cette famille de peintres semble donc se spécialiser, à sa manière, pour un public de Bohême. Très souvent attribuée à un membre autrichien de l'atelier de Johann Gottfried Auerbach, une peinture aujourd'hui conservée à la Galerie nationale de Budapest (P 46) est également liée à un artiste hongrois du nom de Sámuel Horváth, pour lequel nous ne détenons pas beaucoup d'informations, hormis le fait qu'il fut actif en Hongrie au XVIII siècle $^{154}$. Peut-être fut-il aussi membre de l'atelier d'Auerbach.

\section{Une nouvelle génération de peintres à la fin du règne}

$\mathrm{Au}$ moment de la disparition de Meytens et de la réforme académique, les peintres de cour de la dernière décennie du règne, à l'exemple d'Anton von Maron et de Joseph Hickel, proposent des portraits d'apparat toujours imprégnés de pompe baroque $^{155}$ mais aussi d'influence plus bourgeoise et simple, révélant une nouvelle conception du pouvoir et du rôle attribué au dirigeant.

Nombreux sont les peintres qui participent à la réalisation des portraits de Marie-Thérèse et de son empereur et corégent, Joseph II, et qui sont rémunérés par la cour pour exécuter des portraits de la souveraine. Les différentes phases de la vie personnelle et politique de Marie-Thérèse, toutes deux fortement liées, suscitent en effet la production de nouveaux portraits. Jusqu'à sa propre mort

151 Schulze, «Auerbach, Johann Gottfried ».

152 Schulze, « Auerbach, Johann Karl».

153 Fleischer, Das kunstgeschichtliche Material, p. 134: « dem Mahler Auerbach für verschiedene in das Schlohs nach Prag verfertigte Portrait ».

154 Szinyei, Die ungarische Nationalgalerie, p. 121. Voir chapitre III.

155 Ce type de portrait propose un modèle aux côtés des couronnes. 
en 1770, le peintre officiel Martin van Meytens se charge de représenter MarieThérèse veuve. La représentation la plus connue se trouve aujourd'hui au Palais Trautson à Vienne $(\mathrm{P} 180)^{156}$. Parmi les peintres qui réalisent de multiples portraits de Marie-Thérèse, certains comme Anton von Maron, sont influencés par de nouvelles tendances artistiques, qui annoncent déjà le règne de Joseph II.

Après 1765, date de la mort de son époux François Étienne, désormais peinte en habits de veuve, Marie-Thérèse n'accorde, semble-t-il, jamais plus la confiance qu'elle avait accordée à Meytens et à Liotard à un autre peintre. Peutêtre a-t-elle alors moins le goût pour les longues séances de pose. Il lui arrive d'émettre des doutes sur la capacité d'Anton von Maron à réaliser avec succès son portrait, comme en témoignent certains extraits de sa correspondance.

Dans une lettre adressée à l'archiduc Ferdinand datée de 1772, MarieThérèse exprime ses doutes :

J'ai tant à écrire en France et en Italie, que je ne peux être fort courte ; outre cela le peintre Maron est ici, et je me fais peindre de lui pour vous, si cela réussit ; j'en doute un peu encore. Embrassez votre chère épouse de ma part [. . . $]^{157}$.

Toutefois, une telle remarque isolée n'est pas forcément représentative, ni significative, et peut être due à la lassitude de la souveraine âgée, peu satisfaite de son apparence.

Dans d'autres lettres échangées entre le baron de Neny et le baron de Saint Odile à Rome, la souveraine semble particulièrement satisfaite des ouvrages de Maron concernant les portraits des membres de sa famille :

Vous m'y dites d'abord que le Sieur Maron conserve toujours la Mémoire des témoignages de la Satisfaction de Sa Majesté, qui lui furent envoyés de novembre de l'année 1770, pour le tableau représentant Madame l'archiduchesse Grand-Duchesse et sa famille ${ }^{158}$.

156 Voir le portrait de Marie-Thérèse veuve réalisé par Martin van Meytens aux environs de 1765. La souveraine est représentée assise. Sa main droite est posée sur une couronne (probablement une couronne héraldique) représentée à côté de l'insigne de Saint Étienne. Cette peinture à huile est conservée au Palais Trautson à Vienne. Le Palais Trautson est le siège de la garde hongroise créée par Marie-Thérèse (actuel ministère de la Justice).

157 Arneth, Briefe der Kaiserin Maria Theresia, vol 1, pp. 140-141, p. 141.

158 Voir l'Italienische Korrespondenz 1772-75, HHStA AKA 35-5 Italienische Korrespondenz 1772-1775; Lettre du Baron de Neny à Monsieur le Baron de Saint Odile à Rome, De Schönbrunn le 4 May 1772. Concernant trois tableaux du Sieur Maron.

Dans une lettre du 7 Mai 1772, il est écrit « Le tableau de feu Sa Majesté l'Empereur par le Sieur Maron, nous est parvenu hier en parfaitement bon Etat. Notre auguste Souveraine en est enchantée, et va le faire placer tout de suite dans le cabinet de Laiq [...] ».

Dans une lettre datée du 24 juin 1772 adressée par Maron lui-même au baron de Neny. En haut de la lettre, il est précisé que cette lettre a été rapportée à Sa Majesté l'Impératrice et Reine le 10 Juillet suivant. 
Tous ces extraits évoquent certes les portraits de Marie-Thérèse, mais aussi ceux des siens (parents et amis) qui sont tout aussi importants à ses yeux.

Le 4 mai 1772, une lettre du baron de Neny au baron de Saint Odile, écrite à Schönbrunn, plaide en faveur du peintre Maron afin que ce dernier soit payé le plus vite possible :

Sa Majesté fera acquitter aussi cette dette en même temps avec ce qu'elle fera donner au Sieur Maron pour le portrait de feu Sa Majesté l'Empereur qui doit nous parvenir demain, je vous prie donc cher ami de tacher de savoir adroitement ce qui en est, et de m'en informer le plutôt qu'il se pourra pour la direction ultérieure de l'Impératrice et Reine.

Le 24 juin de la même année, une lettre de Maron lui-même, écrite de Rome, adressée au baron de Neny et rapportée à la reine le 10 juillet, rend compte des portraits que Maron doit encore livrer:

Sa Majesté l'Impératrice Reine est pleinement informée au sujet de la quantité de mes ouvrages déjà trop arrierrés et de devoirs qui m’obligent à fixer mon séjour à Rome.

Pour résumer, durant les dernières décennies du règne, entre 1765 et 1780, les principaux peintres actifs à et pour la cour sont en effet Maron et Hickel. Anton von Maron réalise plusieurs portraits du corpus, sans compter les nombreuses copies de ces portraits ; certains portraits de Marie-Thérèse sont en effet attribués à Maron mais sans garantie ${ }^{159}$. Les tableaux de ces artistes, généralement d'assez grandes dimensions, reflètent les nouvelles images du pouvoir en offrant parfois le portrait d'une veuve assise à sa table de travail. Cette image de Maron influence beaucoup l'image que l'on se fait de Marie-Thérèse dans les années 1770 , celle d'une souveraine assidue au travail.

Penchons-nous un peu plus sur cet artiste : Anton von Maron (1733-1808) est un élève de l'Académie viennoise et notamment de Daniel Gran. Il part très tôt pour Rome et devient membre de l'Académie romaine dès $1766^{160}$. Au cours des années 1772 et 1773, lors de sa période de corégence avec Joseph, MarieThérèse fait réaliser par Maron des portraits qui la montrent assise à sa table de travail. Ses commandes royales les plus importantes débutent dans les années 1770. Un portrait demandé juste après la mort de François Étienne en 1765 a parfois été attribué à Maron. Marie-Thérèse y est peinte en veuve, dans la pos-

159 En Slovaquie, le Musée Červený Kameň en possède également un. Enfin, un portrait de Marie-Thérèse assise tenant un papier dans la main gauche, peinte à côté de la couronne impériale familiale, est conservé au Musée régional de Ptuj en Slovénie.

160 Schmittmann, «Maron, Anton von » et Anton von Maron, p. 439. Concernant les commandes de portraits de la maison impériale, voir les pages 42-44 de la biographie de Schmittmann, Anton von Maron. 
ture de fondatrice du chapitre des dames nobles d'Innsbruck. Il s'agit d'une huile sur toile de grandes dimensions avec en arrière-plan une vue sur les montagnes et la ville d'Innsbruck. Le portrait est aujourd'hui conservé à la Hofburg d'Innsbruck comme un prêt du Damenstift (P 174, Figure 2) ${ }^{161}$.

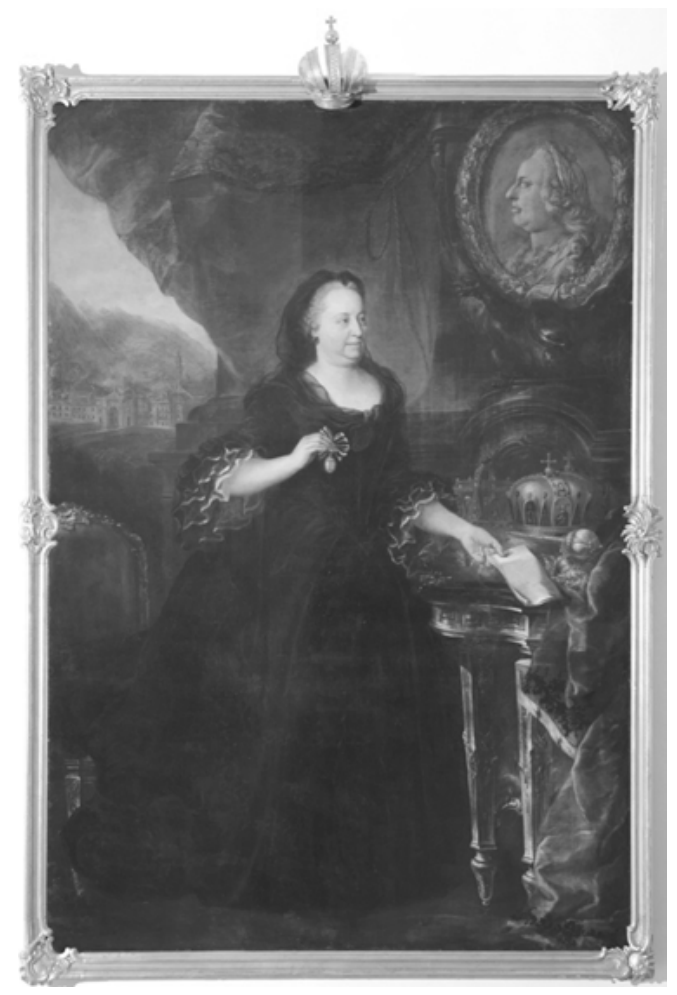

Figure 2: Attribué au peinte Anton von Maron, mais provient probablement d'un autre peintre autrichien, Marie-Thérèse veuve en fondatrice de l'Ordre des Dames nobles d'Innsbruck, après 1765, 230 x $161 \mathrm{~cm}$ @ Burghauptmannschaft Österreich, Sammlung:

Bundesmobilienverwaltung, Hofburg Innsbruck, MD 060828/000, Autriche, Photo: Bunge (Firma Neubauer).

161 Reinwetter, « Maria Theresia als Gründerin des Innsbrucker Damenstiftes »; Trapp, Maria Theresia und Tirol, p. 90. Schmittmann, « Maron, Anton von » et Anton von Maron, p. 439. Ce tableau est attribué par Reinwetter à Maron mais sans garantie, Schmittmann parle même d'une attribution du portrait à Maron qui ne peut pas être retenue. Dans la liste d'inventaire de la Hofburg d'Innsbruck, il n'y a que cette inscription « peintre autrichien après 1765 ». 
Dès 1770, Maron peint à Florence des portraits de la famille grand-ducale de l'archiduc Léopold destinés à la cour de Vienne ${ }^{162}$. Lors de son retour à Vienne en 1772, avec son ami, le peintre paysagiste Joseph Rosa, qui est nommé directeur de la Galerie de peinture impériale à Vienne par Marie-Thérèse en 1772, Maron entreprend un inventaire, ou révision, des tableaux conservés à Vienne et dans les résidences de Prague et Presbourg ${ }^{163}$. À Vienne, en 1772, il entame aussi l'exécution du grand portrait de Marie-Thérèse, du défunt François $\mathrm{I}^{\mathrm{er}}$, ainsi que celui de Joseph $\mathrm{II}^{164}$. L'esquisse de la composition d'ensemble (Vienne, Kunsthistorisches Museum) est probablement déjà exécutée ou terminée à Rome ${ }^{165}$. Maron peint en 1773 le grand et célèbre portrait de la souveraine, assise à sa table de travail, qui est ensuite envoyé à Vienne, aujourd'hui au Kunsthistorisches Museum (P 173, Figure 39). On sait que la souveraine apprécia beaucoup ce tableau et qu'à la suite de cela Maron fut élevé dans le rang de la noblesse ${ }^{166}$.

Faisant partie de cette génération d'artistes autrichiens envoyés à Rome, qui y résident davantage qu'à Vienne, Anton von Maron n'est pas le seul peintre de cette période à exécuter des portraits de Marie-Thérèse et à la peindre sous un jour nouveau. Joseph Hickel, élève de Martin van Meytens, est un autre artiste qui représente la souveraine dans des attitudes similaires à celles rencontrées dans les portraits de Maron, d'une manière déjà novatrice et imprégnée de certains idéaux des Lumières. Né en Bohême en 1736, c'est à Vienne qu'Hickel termine sa vie en $1807^{167}$. Il est tout d'abord l'élève de son père, un artiste de Bohême resté méconnu, puis entre à l'Académie de Vienne afin de parfaire sa formation. Au sein de l'institution viennoise, il se spécialise dans le genre du portrait avant d’être envoyé dans les années 1760 en Italie. À Milan,

162 Schmittmann, Anton von Maron, p. 39.

163 HHStA AKA 35 5, Italienische Korrespondenz 1772-75, « à Son Excellence Monsieur le Comte de Rosenberg à Rome, Schönbrunn le 27 juillet 1772, Concernant l'arrivée de M. Maron à Vienne, Le départ de Monsieur l'ambassadeur Comte de Mahoni de Vienne, Les cours de Milan et de Parme », « [. . . ] On l'occupera ensuite à l'arrangement de notre gallerie de peintures à Vienne, à la révision des Tableaux qui se trouvent dans les châteaux de Prague et de Presbourg [. . .] »; dans une autre lettre adressée à St Odile concernant les occupations de Mr. Maron à la Cour de Vienne, du 6 aout 1772, il est fait mention de "l'état déplorable » de la galerie de Vienne ; Schütz, « Anton von Maron », pp. 324-326.

164 Schmittmann, Anton von Maron, pp. 39-40.

165 Schmittmann, Anton von Maron, pp. 39-40. L'auteur souligne que le peintre garde son logement romain durant cette période et termine les tableaux dans la capitale romaine, bien qu'il ait passé aussi beaucoup de temps à Vienne en 1772.

166 Voir Prohaska, "Anton von Maron », p. 453; Schütz, " Anton von Maron », pp. 324-326. Sur le portrait, voir note 395, chapitre X. Voir aussi Schmittmann, Anton von Maron, p. 40, pp. 283-288, pp. 287-288.

167 Lechner, « Hickel »; Popelka, « Hickel ». 
Parme et Florence ${ }^{168}$, Hickel réalise des portraits de grandes personnalités de l'époque, comme par exemple des membres de la famille des archiducs Léopold et Ferdinand, commandés par la souveraine Marie-Thérèse. En 1769, il fait partie de l'Académie de Florence ${ }^{169}$. Deux ans plus tard, il est nommé KaiserlichKöniglicher Kammermaler. Dans le cadre de cette activité, il travaille des le $1^{\mathrm{er}}$ octobre 1772 comme Galerie Adjunkt à la galerie viennoise pour un solde de cour de sept cents florins par $\mathrm{an}^{170}$. Comme d'autres peintres tels Meytens, Joseph Hickel est le portraitiste des membres de la famille impériale mais aussi des ministres, des hauts ecclésiastiques, de la noblesse, des généraux ainsi que des acteurs du théâtre national ${ }^{171}$. Il réalise ainsi des portraits notamment pour Joseph Wenzel prince de Liechtenstein, aujourd'hui à l'Albertina de Vienne ${ }^{172}$. Lorsqu'il commence à travailler à partir de 1765 pour la cour de Vienne, la souveraine lui confie très vite des missions très honorifiques et importantes comme l'exécution des portraits individuels de ses propres enfants ${ }^{173}$.

Hickel peint un portrait de Marie-Thérèse dont le style évoque déjà un changement entre le portrait baroque et une conception plus bourgeoise du portrait de cour, comme on peut également le remarquer dans la manière de peindre d'autres portraitistes royaux de la même époque ${ }^{174}$. Ce portrait est aujourd'hui conservé dans la Maria-Theresien-Saal du Musée de l'histoire de l'Armée à Vienne. Cette huile sur toile est de grandes dimensions comme la plupart des tableaux de représentation traditionnels (P 156, Figure 42). Marie-Thérèse veuve, est peinte en pied, debout, près d'une table recouverte de livres, de papiers et d'un encrier. Walter Pillich recense pour l'année 1777 un portrait de Marie-Thérèse der regierenden Röm.Kais. Maitt pour lequel le peintre Joseph Hickel, reçoit quatre cents Gulden ou florins ${ }^{175}$.

Dans les années 1760 et 1770, plusieurs artistes sont encore cités dans les Kammeralzahlamtsbücher, les livres des comptes de la Chambre. Une nouvelle génération arrive. Johannes Tusch (1738-1817) est l'un d'entre eux ; ce peintre et miniaturiste devient dès 1772 pensionnaire de l'Académie impériale et royale à Rome. Un certain nombre de ses portraits se trouvent aujourd'hui au Kunsthisto-

168 Leistner, Joseph Hickel, p. 4; Thomasberger, « Joseph und Anton Hickel », p. 7 et pp. 24-28.

169 Thomasberger, « Joseph und Anton Hickel », p. 7.

170 Pillich, 36. Hofparteienprotokoll 1772, vol. 19, p. 512, 699. 11 Octobre 1772 Vienne.

171 Leistner, Joseph Hickel, p. 8; Thomasberger, « Joseph und Anton Hickel ».

172 Thomasberger, «Joseph und Anton Hickel », p. 16, figure 2.

173 Thomasberger, « Joseph und Anton Hickel », pp. 31-38; Leistner, Joseph Hickel, pp. 10-13, 10.

174 Leistner, Joseph Hickel, pp. 11-14, 13-14, figure 7, p. 11; Thomasberger, « Joseph und Anton Hickel », pp. 39-41, figure 19.

175 Pillich, 39. Hofparteienprotokoll 1777, vol. 19, p. 521, 766. 17 Février 1777 Vienne. 
risches Museum de Vienne, comme dans les collections de la famille Liechtenstein (P 175) ${ }^{176}$ ainsi qu'au château de Feldsberg, où est conservé un portrait de la souveraine en veuve ${ }^{177}$. Johannes Tusch obtient la place de conservateur dans la galerie de tableaux en 1776 pour une rétribution de sept cents florins par $\mathrm{an}^{178}$.

En fin de règne, les portraits royaux sont toujours aussi nombreux. Le portrait de la souveraine devient alors plus populaire que jamais. C'est également la marque d'une certaine forme de légitimité de la personne royale. Après la mort de Meytens, les artistes sont moins centrés autour d'un atelier comme celui de Meytens, atelier qui n'a d'ailleurs jamais eu de monopole - même si le portrait que Meytens et son atelier réalisent de Marie-Thérèse a certainement influencé les générations suivantes. À partir des années 1760, plusieurs peintres sont alors employés pour diffuser l'image de Marie-Thérèse veuve, vêtue de noir. Certains peintres disposent encore d'un nom comme Anton von Maron. Ces peintres sont des académiciens mais passent souvent plus de temps à Rome qu'à Vienne.

Antonio Pencini (Bencini) est un autre artiste très souvent mentionné dans les registres de comptes durant la dernière décennie du règne. Hof- und Kammermaler, actif à Vienne depuis 1753, il est recensé dès 1741 comme élève de l'Académie de Vienne. Il peint un certain nombre de portraits en miniature de Marie-Thérèse veuve, du prince Charles de Lorraine, des archiducs Joseph, Léopold, Maximilien, Ferdinand, des archiduchesses Marie-Caroline, Marie-Antoinette, Marie-Amélie, Marie-Élisabeth, Marie-Christine et Marianne ${ }^{179}$. Chaque trimestre à partir de juin 1772, il est censé réaliser des portraits de Marie-Thérèse et de son fils Joseph II. Ce peintre a donc un contrat régulier avec la cour, comme Philipp Ferdinand von Hamilton plusieurs décennies plus tôt ${ }^{180}$. Il exécute également en 1765 deux portraits de la souveraine et deux portraits de son fils Léopold, grand duc de Toscane ${ }^{181}$, puis il livre en 1770 deux portraits de Marie-Thérèse, tout comme en 1771 et $1772^{182}$.

176 Un grand portrait de Marie-Thérèse veuve, conservé dans les collections Liechtenstein à Vienne est attribué au peintre Johannes Tusch. Peinte à côté de la couronne de Hongrie et du chapeau archiducal, la souveraine, vêtue de l'habit de veuve, dirige son doigt vers le buste de son défunt époux, tandis qu'elle arbore sur sa poitrine l'insigne de l'Ordre de Saint-Étienne.

177 Thieme Becker, vol. 33/34, p. 503; Garas, Barokk festészet.

178 Pillich, 38. Hofparteienprotokoll 1775, vol. 19, p. 520, 757. 11 Novembre 1776 Vienne.

179 Thieme Becker, vol. 25/26, p. 374. Haupt, Das Hof-und hofbefreite Handwerk, p. 230, un certain Johann Anton Pencini est mentionné, mort en 1776, « Hof-und Kammermaler, kais. Miniaturmaler ». Lechner, « Porträtmalerei », p. 51.

180 Fleischer, Das kunstgeschichtliche Material, p. 129.

181 Fleischer, Das kunstgeschichtliche Material, p. 94.

182 Fleischer, Das kunstgeschichtliche Material, pp. 131-133. 
$\mathrm{Au}$ cours de la dernière décennie du règne, le peintre viennois Anton Somos est recensé dans les registres de comptes de la cour. Il est rémunéré en 1774 pour trois portraits de Charles VI, Marie-Anne et Marie-Thérèse, et en 1775 pour un portrait de la famille impériale ${ }^{183}$. Autre peintre autrichien, Johann Michael Millitz (1725-vers 1784), également peintre de cour actif à Vienne, est immatriculé à l'académie en 1747 comme élève de Martin van Meytens. Millitz peut être considéré comme un successeur de Meytens, particulièrement remarquable et remarqué pour la qualité et la minutie de ses portraits ${ }^{184}$. Ayant beaucoup peint pour les noblesses autrichienne, tchèque et hongroise, Millitz semble très proche de la souveraine comme en témoigne la correspondance de Marie-Thérèse, notamment dans cette lettre adressée à l'archiduchesse Marie-Christine qui était alors en voyage et manifestement accompagnée de Millitz : « Le 25 janvier de l'an 1776. Ma chère fille. Tout ce que vous me dites de votre agréable situation, me fait un vrai plaisir. [. . .]. Que dit Millitz, se plaît-il à quelques beautés de peintures, de sculptures ? " ${ }^{185}$. Aujourd'hui, un de ses portraits, sur lequel Marie-Thérèse figure en veuve, est exposé à la Galerie nationale de Budapest. Ce portrait est une huile sur toile représentant Marie-Thérèse jusqu'au genou, probablement peinte vers 1770. En arrière-plan est représenté le défunt empereur François Étienne (P 155).

Durant les deux dernières décennies du règne, le peintre Hubert Maurer (ou Mauerer) (1738-1818) propose un portrait de Marie-Thérèse dans le même style que ceux de Maron et d'Hickel, avec draperies et statues en arrière-plan. Élève du peintre de la cour de Bavière, Johann Georg Winter, et du célèbre sculpteur Franz Xaver Messerschmidt, Hubert Maurer devient après 1762 membre de l'Académie viennoise. Ce peintre est représentatif de cette nouvelle génération d'artistes, notamment de peintres et portraitistes, que Vienne, notamment Marie-Thérèse et Kaunitz, envoie se former à Rome à partir de $1772^{186}$. Si ses premières œuvres rappellent fortement la peinture baroque, ses œuvres ultérieures rendent davantage compte des tendances classiques de son époque ${ }^{187}$. Quelques-uns de ses portraits sont exposés à Salzbourg mais aussi dans la salle du Palais de l'Académie de Mantoue comme les trois portraits de Marie-Thérèse, de François Étienne et de Joseph II datant de 1770.

183 Fleischer, Das kunstgeschichtliche Material, p. 143, 145; Garas, Magyarországi barokk festészet, vol. 2, p. 252.

184 Buzási, « Johann Michael Millitz »; Garas, Magyarországi barokk festészet, vol. 2, p. 237.

185 Arneth, Briefe der Kaiserin Maria Theresia, vol. 2, pp. 391-392.

186 Czeike, Historisches Lexikon Wien, vol. 4, p. 208; Goldschmidt, « Maurer ».

187 Il aurait entre autres peint deux portraits de Marie-Thérèse et de François Etienne, aujourd'hui à l'abbaye de Wilten ; voir Hammer, Die Paläste und Bürgerbauten Innsbrucks, pp. 161-162. 
Après sa mort, un de ses élèves et ami, Johann Michael Sattler, rédige sa biographie dans laquelle il mentionne que Maurer a peint d'autres portraits des membres de la famille impériale. Ainsi le baron Joseph Sperges, alors président de l'Académie impériale, commande auprès du peintre trois portraits en pied : un portrait de sa Majesté l'empereur François $\mathrm{I}^{\mathrm{er}}$, un autre de Marie-Thérèse (P 227) et enfin de l'empereur Joseph pour l'Académie de Mantoue ${ }^{188}$. Une fois les modèles terminés, le baron Sperges les montre au prince de Kaunitz qui les admire et demande à l'artiste de réaliser son portrait, en pied, revêtu de l'habit de la Toison d'Or' ${ }^{189}$.

Le prince de Kaunitz commande également à Maurer un portrait de MarieThérèse pour l'envoyer à la cour impériale russe à Saint-Pétersbourg. La souveraine exauce la demande de Kaunitz en posant pour le peintre. Comme nous l'avons déjà mentionné, cette commande donne lieu à une anecdote intéressante concernant la relation entre Marie-Thérèse et ses peintres. Alors qu'une table manque pour soutenir la souveraine en train de poser, elle aide elle-même le peintre à en porter une de la pièce voisine ${ }^{190}$. Ce tableau est, aux dires de Sattler, aussi bien réussi que les autres travaux pour lesquels Maurer s'était déjà fait connaître ${ }^{191}$.

Un peu plus tard, Maurer sera le premier pensionnaire envoyé à Rome par l'Académie de Vienne, sur la recommandation de Kaunitz. En rentrant à Vienne après un séjour de plusieurs années à Rome, il présente au chancelier d'État les tableaux et les études qu'il y a réalisées. Toujours selon Sattler, Kaunitz aurait exprimé et rapporté à la souveraine son enthousiasme devant les progrès faits par l'artiste, à la suite de quoi celui-ci est invité à exécuter de nouveau des portraits de MarieThérèse et de Joseph II, destinés cette fois à l'université de Pavie ${ }^{192}$.

188 Les œuvres évoquées sont actuellement exposées dans le hall de la salle Piermarini du Palazzo Accademico à Mantoue, ces peintures sont la propriété de l'Académie Virgiliana. L'architecte Paolo Pozzo a conçu la salle Piermarini dans le but d'accueillir ces trois portraits qui avaient été déjà apportés de Vienne. Les dirigeants de l'Académie se rassemblaient dans cette pièce, située au premier étage du théâtre académique scientifique, créé par Antonio Bibiena. Le hall, construit entre 1773 et 1775, exposait les trois grands tableaux peints à Vienne en 1770 par Hubert Maurer ainsi qu'une dédicace spéciale pour Marie-Thérèse. Merci à Chiara Pisani pour ces informations concernant Mantoue. Voir aussi chapitre IV.

189 Sattler, Lebensgeschichte, pp. 47-48.

190 Sattler, Lebensgeschichte, pp. 48-49, p. 49.

191 Sattler, Lebensgeschichte, pp. 49-50.

192 Sattler, Lebensgeschichte, pp. 87-88: «Verzeichnis der bekannten gemahlten Bilder von Hubert Maurer als Porträts: 2: Kaiser Franz, Maria Theresia und Kaiser Joseph in Lebensgrösse nach Mantua; 5: Die Kaiserinn Maria Theresia nach St Petersburg; 6: Die Kaiserinn Maria Theresia und Kaiser Joseph, in Lebensgrösse nach Pavia »; Sattler, Lebensgeschichte, pp. 53-54, 69-70. 
Enfin, évoquons le peintre Johann Joseph Hauzinger qui fréquente l'Académie viennoise sous Jacob van Schuppen, Paul Troger et Daniel Gran. Kammermaler en 1761, il devient par la suite professeur de peinture d'histoire à l'Académie de Vienne. Hauzinger exécute des portraits royaux pour l'université hongroise de Trnava (P 241), ainsi que de nombreux portraits de la famille impériale pour Vienne, Presbourg (P 225) et Innsbruck entre 1773 et $1777^{193}$.

Pour résumer, durant une assez longue période, ce sont des peintres d'origine étrangère, tels Martin van Meytens, Andreas Möller, ou encore l'ami proche de Marie-Thérèse, le peintre Jean-Étienne Liotard, qui semblent emporter les faveurs de la cour, même si les peintres de cour autrichiens sont loin d'être absents. Il existe une certaine imitation entre les portraits royaux et les portraits aristocratiques. Les commandes de portraits sont déterminées en partie par le goût d'une aristocratie que l'on pourrait qualifier de viennoise mais qui reste quand même très marquée par le cosmopolitisme propre à la Monarchie. Il s'agit entre autres des grandes familles nobles qui conservent des attaches dans les divers pays de la Monarchie, comme en atteste la popularité des portraits réalisés par Martin van Meytens au sein des provinces de l'empire. Parmi les personnalités issues de pays étrangers se rendant à Vienne, mentionnons par exemple le Français, d'origine lorraine, Joseph Ducreux (1735-1802), qui réside en 1769 à Vienne et peint des portraits de Marie-Thérèse veuve qu'il rapporte ensuite à la cour de France pour la reine Marie-Antoinette ${ }^{194}$. Au sein des portraitistes étrangers qui travaillent à la cour de Vienne, dont ils sont à l'occasion les invités, figure aussi le peintre genevois Jean-Étienne Liotard (1702-1789), également nommé le peintre turc en raison de son long séjour dans l'Empire ottoman. Liotard a une prédilection pour les peintures au pastel alors que Meytens emploie la peinture à huile pour une représentation officielle, classique, et en majesté de la souveraine. Au moyen de cette technique de peinture, Liotard livre une image plus intimiste et familière de la souverain $\mathrm{e}^{195}$.

Parmi les plus belles œuvres du peintre figurent les portraits des Habsbourg-Lorraine ${ }^{196}$. Gagnant très vite l'amitié de la souveraine, le succès du pein-

193 Garas, Magyarországi barokk festészet, vol. 2, p. 222; Rudolf, Ulreich, Karpatendeutsches Biographisches Lexikon, p. 123; Papco, Rakúsky barok, vol. 2, p. 516.

194 Voreaux, «Ducreux ».

195 Bleeker, «Le pastel »; Loche, « Bildnis eines nüchternen Malers », p. 9.

196 Roethlisberger, Loche, Liotard, vol. 1, pp. 18-51, 299-320. Né à Genève en 1702, Liotard se rend à Rome en 1736 puis part pour Constantinople. Le peintre séjourne à Vienne trois fois, d'abord de 1743 à 1745 . Il réalise des portraits des grandes personnalités de l'époque qu'il peint notamment en costume ottoman, c'est le cas de Marie-Thérèse et de certaines de ses filles, comme Marie-Christine. Peintre de pastel principalement, il trouve certainement là un terrain d'entente avec la souveraine qui, elle-même, avait été initiée dans son enfance à cet art 
tre genevois à Vienne est immédiat, et ce tout au long du règne de MarieThérèse. Ses principaux portraits de Marie-Thérèse la montrent sous un type similaire (P 149, P 150, P 151) : la tête est dessinée de la même manière, de dimensions identiques ${ }^{197}$. Les portraits de Liotard sont plus petits que ceux de Meytens ou Auerbach. Limités à la demi-figure, ils laissent moins de place aux insignes royaux $^{198}$, même si ces symboles ne sont pas totalement absents. On peut surtout remarquer la relative beauté de Marie-Thérèse dans les portraits peints par Liotard. Par rapport aux nombreux portraits réalisés par Meytens et son atelier, les portraits de Liotard sont plus intimistes, intimes, et familiers. Ils ont un caractère plus privé ${ }^{199}$, moins conventionnel et majestueux. Les insignes du pouvoir sont moins mis en avant dans ses tableaux que dans ceux de Meytens. Dans les portraits de Liotard, la représentation du corps naturel de Marie-Thérèse est soulignée par rapport à son corps politique, la souveraine apparaît ainsi plus proche et accessible aux spectateurs. Même lorsque les insignes du pouvoir sont peints, l'attention du spectateur est davantage attirée vers le personnage, notamment vers son visage, que vers ses attributs. Les œuvres de Liotard sont, comme celles de Meytens, très souvent recopiées tout au long du règne. Les tableaux du peintre suisse sont peut-être mieux adaptés à des demeures privées que les grands portraits de cour ${ }^{200}$. Même s'il exécute aussi des tableaux de Marie-Thérèse qui entretiennent des rapports étroits avec les propres portraits de Meytens, l'artiste suisse simplifie souvent les tableaux, en diminuant notamment les dimensions. En tant que peintre indépendant, Jean-Étienne Liotard est un peu plus libre dans son interprétation et dans sa composition de la figure royale, laquelle peut alors apparaître plus proche et réaliste au spectateur ${ }^{201}$. Par ailleurs, Marie-Thérèse entretient un rapport plus familier avec Liotard, avec lequel elle passe volontiers du temps pour poser ${ }^{202}$, ce dont se serait d'ailleurs plaint Meytens. Cela peut s'expliquer par le fait que Liotard n'est que de passage à Vienne ${ }^{203}$. Les portraits royaux réalisés par Liotard servent aussi de modèles originaux à d'autres peintres,

par l'artiste Rosalba Carriera. Sur sa vie et ses œuvres, voir aussi Fosca, La vie ; Koos, « Liotard »; Loche, « Bildnis eines nüchternen Malers »; Loche, « Liotard ».

197 Roethlisberger, Loche, Liotard, vol. 1, p. 301 : « [. . . ] il est évident que Liotard travailla à partir d'un seul modèle de la tête qu'il répéta chaque fois au millimètre, variant les vêtements selon les instructions de la souveraine [. . . ]».

198 Roethlisberger, Loche, Liotard, vol. 1, p. 302.

199 Roethlisberger, Loche, Liotard, vol. 1, p. 302.

200 Roethlisberger, Loche, Liotard, vol. 1, p. 302.

201 Liotard a écrit un traité de peinture ; voir Liotard, Traité des principes et des règles de la peinture.

202 Koschatzky, « Jean-Etienne Liotard », voir entre autres p. 315.

203 Sur les séjours de Liotard à Vienne, voir aussi Fosca, La vie, pp. 28-29, 88-89, 105-112. 
comme l'atteste l'exemple du peintre flamand Matthias de Visch à Bruges (P 124, Figure 11) qui s'inspire certes de portraits de Meytens mais aussi de ceux de Liotard pour exécuter différentes répliques du portrait royal destinées à l'hôtel de ville de Bruges.

\section{Conclusion}

De cette analyse des peintres qui ont travaillé à la cour ou du moins à Vienne durant les quatre décennies du règne, résulte l'image d'un réseau assez complexe et hétérogène, même s'il gravite autour d'une figure centrale, Meytens. Le peintre d'origine suédoise Martin van Meytens, et son atelier, disposent en effet d'une véritable position sociale qui leur assure un certain renom. Si Meytens, peintre réputé en Autriche, comme en Europe, occupe sans conteste une place à part et déterminante pour l'image de Marie-Thérèse, notamment vis-à-vis de la postérité, d'autres peintres sont également très actifs et produisent de nombreux portraits royaux, dont la composition évolue au cours des quarante années de règne. Divers peintres de cour ne cessent en effet de réaliser et de reproduire des images de la souveraine. Certains, comme Liotard, jouissent d'une plus grande liberté, ce qui ne les empêche pas d'être productifs. Toutefois, ils ne sont pas les seuls car des peintres provinciaux et locaux participent également activement à la représentation impériale. 